\title{
A Study on the Current Situation and Protection of Zhoushan Folk Ocean Paper-Cut Art
}

\author{
Yu Lihong*, Song Qiuqian, Chen Shiding \\ Zhejiang Ocean University, Zhoushan, China
}

*Corresponding Author: Yu Lihong, Zhejiang Ocean University, Zhoushan, China

\begin{abstract}
Zhoushan folk ocean paper-cut is an art with distinctive ocean characteristics and unique style in Chinese paper-cut art, which conveys the material and spiritual life of the island people. Due to many realistic conditions such as the changes of people's lifestyle and aesthetic taste, Zhoushan folk ocean papercut is faced with many difficulties, but as the fifth batch of "intangible cultural heritage" of Zhoushan, ocean paper-cut has also gradually developed. This paper analyzes the current status of Zhoushan folk ocean papercut art, and puts forward protection and countermeasures from three perspectives of government, market and inheritors.
\end{abstract}

Keywords: Zhoushan; Ocean paper-cut; Intangible cultural heritage; Current situation; Protection

\section{INTRODUCTION}

Folk paper-cut art, also known as flower-cut, twisted flowers, window-cut, paper-carving etc., which is a part of traditional Chinese art. It is widely spread with high aesthetic value and embodies the material and spiritual life of the people in all regions of China, containing a long history and rich cultural characteristics of the Chinese nation.

Among them, ocean paper-cut is an art with distinctive ocean characteristics and unique style in Chinese folk paper-cut art, mainly distributed in Qingdao, Zhoushan, Wenling and other coastal areas in Zhejiang Province. Due to the change of people's life style and aesthetic taste, Zhoushan folk ocean paper-cut has encountered difficulties in the development process. In 2013, Zhoushan folk ocean paper-cut art was rated as the fifth batch of "intangible cultural heritage " in Zhoushan city with its unique style. The inheritance and protection of ocean paper-cut has attracted attention from all walks of life. After a long period of protection and development, ocean paper-cut has provided strong support for the development and exchange of regional economy and culture, gradually producing good local culture, economy and social effects.

\section{Characteristics of Zhoushan Folk OCEan Paper-Cut ArT}

At present, whether in the North or in the South, whether in the central plains or in the border areas, whether in the Han nationality or minority areas, most regions of China circulate and spread folk paper-cut. Folk paper-cut of different regions and nationalities shows distinct differences. Most of the Northern paper-cut style is rough, simple; the Southern paper-cut is more delicate, more elaborate and more beautiful, while Zhoushan folk ocean paper-cut has both the roughness of the North and the delicateness of the South paper-cut. Zhoushan folk ocean paper-cut takes ocean culture as its main theme, focusing on the marine regional characteristics of Zhoushan. Its paper-cut has bold imagination. The creation of the paper-cut mainly depend on the coastal people's marine production and life as well as the coastal marine environment. It not only reflects the themes of sea production such as sunning, snail picking, fishing, etc., but also reflects the themes of marine organisms such as fish, turtle, shrimp and crab. It also reflects the themes of marine folk activities such as the Opening Sea Festival and Xieyang Festival -- a festival delivering thanks to the sea. There are also themes reflecting the daily life and marine behavior culture of island residents. With the wide application of aquarium seafood and marine production and life, there are obvious differences between ocean papercut and inland paper-cut, central plains paper-cut. (Bi Xuling, 2016) In addition, there is also ocean paper-cut describing new urban atmosphere that reflects the prosperity of Zhoushan Archipelago New Area. 
Zhoushan ocean paper-cut tools include scissors, carving knives and paper. Paper-cut pays attention to the combination of virtual and real to show the dynamic, vivid scene. Most of its production mostly uses monochrome, mainly red paper, but in order to echo the ocean theme, blue paper also often appears in ocean paper-cut works.

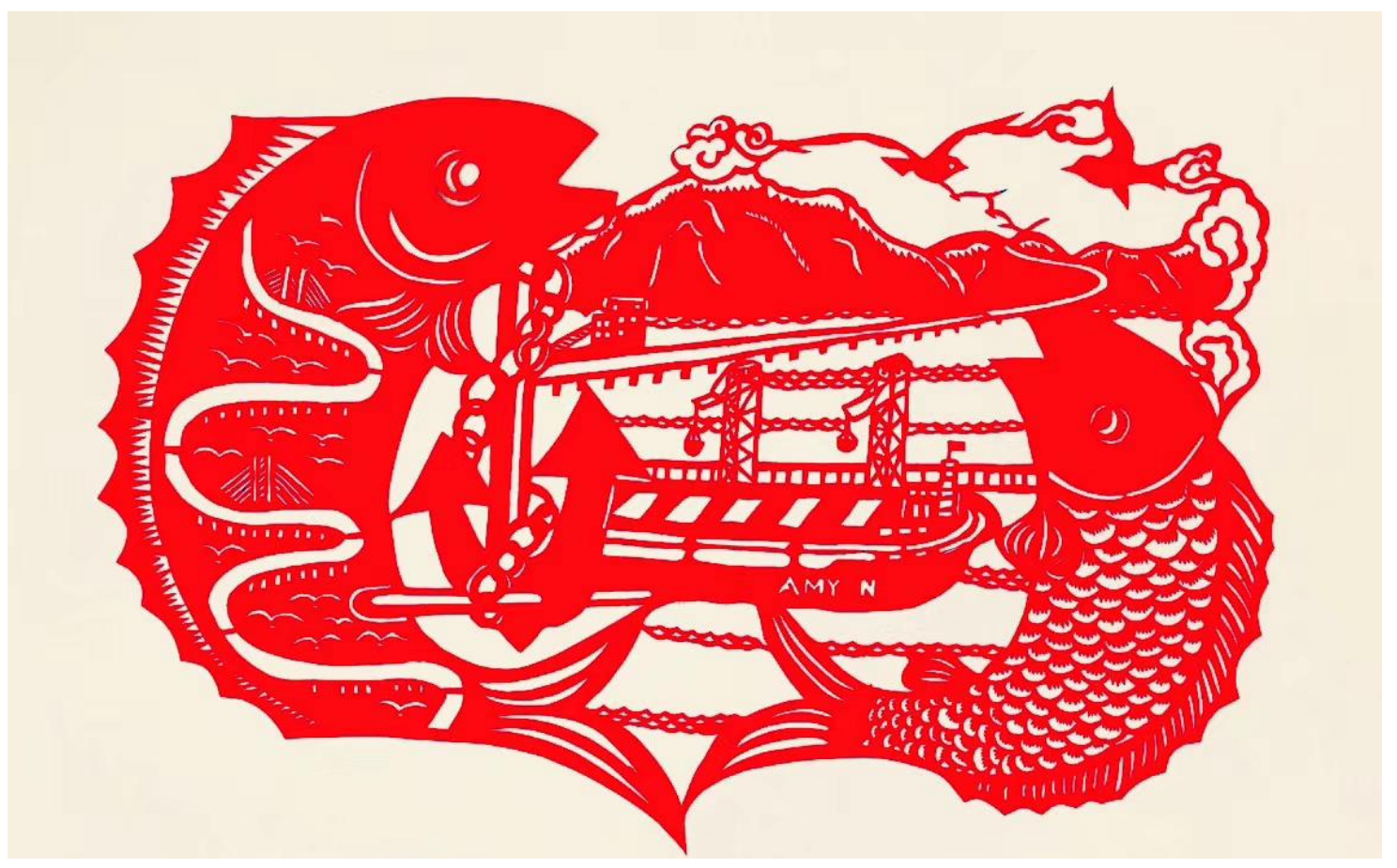

Fig1: Chen Shiding's Work: The Dream of the Sea

\section{Paper-Cut Image and Folklore Connotation of Zhoushan Folk Ocean Paper-Cut ART}

Folk paper-cut image is the embodiment of people's inner thoughts, which contains the folk custom connotation, and reposes people's understanding and wishes for society and life. Zhoushan folk ocean paper-cut also contains the spirit of the people of Zhoushan islands. The harsh natural environment, unpredictable production and living conditions, and the hardships of marine production around Zhoushan islands make the people of Zhoushan revere the sea. They appreciate the harvest of every trip and cherish every harvest, praise the ocean and the brave people who have brought abundant food to the people. Therefore, Zhoushan ocean paper-cut usually shows fishery production activities, such as catching the sea, picking up snails, weaving nets and so on. The rich imagination and delicate composition of Zhoushan paper-cut convey the indomitable pursuit of life of the island people, and their absolute reverence and gratitude for the ocean.

\section{The Survival Status of Zhoushan Folk OCEan Paper-Cut ART}

In the process of development, Zhoushan folk ocean paper-cut art faces multiple difficulties, but also strive to find a way out in the plight. The dilemma of Zhoushan folk ocean paper-cut art mainly includes the aging of paper-cut artists, the decrease of practicability, the unpopularity of patterns and so on. At present, ocean paper-cut also ushered in a new situation of development.

\subsection{The Plight of Zhoushan Folk Ocean Paper-Cut Art}

\subsubsection{Aging of Folk Paper-Cut Artists}

Due to the impact of urbanization and industrialization and lack of policy support, paper-cut artists have to transform to other professions. At present, the folk paper-cut artists are gradually moving towards an advanced age. Physical conditions such as vision, hand flexibility and so on will affect the paper-cut activities.

\subsubsection{Decreased Practicability of Paper-Cut}

The crisis of paper-cut is caused by the change of residents' life style. Nowadays, people's home decoration and printed matter are becoming more and more abundant. Paper-cut has lost its position in 
home decoration during the Spring Festival. In modern life, various decorative printing products and paper decorations are popular because of their various styles. Moreover, the traditional paper-cut has high price as a result of handiwork and poor preservation.

\subsubsection{Unpopular Traditional Patterns}

Influenced by globalization and modern concepts, people's aesthetics has changed. Zhoushan ocean paper-cut is rooted in the islands, attached to marine production and life, and various traditional folk customs, emitting a strong "local flavor" and "fishy taste". Traditional paper-cut patterns can no longer meet the aesthetic taste of modern people. Since most of the people engaged in paper-cut art have entered the middle and old age, their designs are relatively traditional in terms of themes and forms. People in modern society are influenced by Western and global tastes. They consider paper-cut as an old and vulgar thing. Traditional paper-cut patterns have lost their appeal to modern people.

\subsubsection{Lack of Paper-cut Inheritors}

Firstly, under the influence of modern technology and science, people's entertainment is usually mobile phones, computers etc.. Few people kill time by trying paper-cut. Secondly, in the fast-paced modern life, fewer and fewer people are willing to spend time on paper-cut, which requires patience and carefulness. Thirdly, under the modern education which pays attention to scientific knowledge, people mainly accept scientific and cultural knowledge in schools. After-school or weekend art training classes are usually dancing, musical instruments and so on. Paper-cut, as a kind of handicraft art, cannot add points for entrance examination to higher school, which reduces the interest of parents and children in learning, and leads to fewer and fewer people learning paper-cut.

\subsection{Recent Development of Folk Ocean paper-cut Art in Zhoushan}

After Zhoushan folk ocean paper-cut was appraised as the fifth batch of intangible cultural heritage of Zhoushan City, all sectors of society began to pay attention to the protection and inheritance of ocean paper-cut. The government, the market and the inheritors have taken certain measures. Ocean papercut ushered in a new development situation.

\subsubsection{Ocean Paper-cut Entering Schools}

Since being selected as the intangible cultural heritage, Zhoushan Ocean paper-cut has gradually entered the campus. At present, some kindergartens, primary and secondary schools and local universities in Zhoushan Archipelago New Area have set up the course of paper-cutting, or established paper-cutting associations. In the course of ocean paper-cut, the class is taught by papercut teachers or paper-cut artists. Students can feel the rich and colorful ocean culture in the process of paper-cut, exercise their hand operation ability, and experience the charm of intangible cultural heritage.

\subsubsection{Ocean Paper-cut Appearing in Zhoushan Large-scale Activities}

In the large-scale activities held in Zhoushan, such as the International Island Tourism Conference and the China Ocean Culture Festival, the emergence of ocean paper-cut also adds a different color to various activities. Chen Shiding, the inheritor of Shengsi ocean paper-cut, was invited to attend the International Island Tourism Conference. During the event, he presented paper-cut skills to guests from home and abroad and presented them with paper-cut works. The paper-cut works produced by artists on the spot left a deep impression on the audience.

\section{Protection Countermeasures Zhoushan Folk OCean PaPer-cut Art}

In the process of protection and inheritance of intangible cultural heritage, including Zhoushan marine paper-cut, the government, market and inheritors should play their respective roles so as to ensure the sustainable development and continuation of the intangible cultural heritage. In this process, the three should interact effectively.

To be specific, the government should grasp and coordinate from the macro level, and the folk inheritors should start and implement from the micro level. At the same time, driven by the market, the interaction between the government and the inheritor will be more effective. (Wang Ying, 2014) 


\subsection{Government Level}

From the perspective of government, it is the government's responsibility to inherit and protect and develop local "intangible cultural heritage". Local governments should issue relevant policies to protect the development of folk paper-cut art according to the actual needs of folk paper-cut art. On the one hand, the government should introduce policies that are beneficial to folk paper-cut artists. Due to various practical reasons, such as low social status and low income, many folk artists no longer engage in paper-cutting related professions, which gradually leads to the decline of ocean papercutting. This requires the government to implement the existing relevant policies, give certain material and spiritual support to the inheritors, and improve the status of paper-cut artists. On the other hand, the government should strengthen the publicity and education of Zhoushan Ocean paper-cut, an intangible cultural heritage, through various media platforms, and vigorously create a good atmosphere of public opinion for consciously inheriting Zhoushan ocean paper-cut.

\subsection{Market Level}

The industrialization of Zhoushan folk ocean paper-cut is a very complex system engineering, which involves a series of links, including the classification and evaluation of the related resources of papercut, the selection of market demand and the part that can be industrialized, the development and utilization of it, the creation of corresponding products, and the transmission to the market through specific channels for trading profits. (Wen Lei, 2017)

However, in the process of industrialization, we should pay special attention to the following aspects: on the one hand, the value of Marine paper cutting should be explored to promote the protection and development of paper cutting with economic benefits and promote the cultural inheritance of ocean paper-cut art in the contemporary era. On the other hand, attention ought to be paied to the negative impact of paper-cut industrialization on paper-cut, so as to prevent excessive marketization. We cannot only pay attention to economic interests but ignore substantive protection, which neglects the protection of paper-cut art in large-scale production. Although it is conducive to the protection and development of ocean paper-cut, it also destroys the original taste of ocean paper-cut and the authenticity of paper-cut to a certain extent. In the industrialization of Zhoushan folk ocean paper-cut, we should not only pay attention to economic interests, but also take social benefits into account and protect the external natural and cultural ecology of paper-cut.

The industrialization of paper-cut includes selling traditional paper-cut products, developing products of tourism culture, and establishing folk square, etc.

\subsection{Inheritor Level}

In the process of protection and development of ocean paper-cut art, the government, market and inheritors should interact effectively. But among these three, the paper-cut inheritor is the key, and the inheritor should give full play to his subjective initiative.

\subsubsection{Building Cultural Confidence}

From the perspective of inheritors of ocean paper-cut, paper-cut artists should have a sense of cultural confidence, responsibility, positive optimism and innovative spirit. It requires paper-cut inheritors to always have this cultural confidence, promoting local art to the society and the whole country through ocean paper-cutting. In addition, take measures to constantly cultivate the next generation of talents, and spread and inherit ocean paper-cutting.

\subsubsection{Cultivating and Training Talents}

The further inheritance and development of Zhoushan ocean paper-cut art mainly depends on papercut talents. Only with the continuous expansion of talents, can the paper-cut continue to be inherited and innovated. With the support of the government, paper cutting artists should strengthen the publicity of ocean paper cutting to attract more people to learn paper cutting. The main objects are primary and secondary school students, local college students, retirees and social paper-cut enthusiasts. Paper-cut artists can go to schools, communities and other places to spread and impart paper-cut skills through paper-cut public welfare lectures, paper-cut folk activities, paper-cut classes and other ways. 


\subsubsection{Innovating on the Basis of Inheritance}

In order to attract the public's attention, Zhoushan Ocean paper-cut, a treasure of "intangible cultural heritage", must be continuously innovated on the basis of inheritance, so as to meet the modern aesthetic and market needs and improve competitiveness. The innovation of ocean paper-cut can be carried out from the aspects of pattern, material, mounting method and reference of paper-cut works at home and abroad.

The first is the innovation of patterns. At present, ocean paper-cut is mostly based on ocean production activities such as catching up with the sea and drying nets. Ocean paper-cut artists can focus on the beautiful scenery, delicious food, celebrities and famous buildings of Zhoushan to create novel and unique paper-cut works of island. Zhoushan is rich in natural resources, pleasant scenery, delicious food, celebrities, famous architectural paper-cut works close to people's lives, can arouse people's resonance.

Secondly, materials other than paper can be selected for creation. Paper is soft, easy to damage, easy to damp, easy to fade, and not easy to preserve. Paper-cut new materials can choose stickers, magnetic stickers, cotton cloth, etc., will not fade, and stickers, magnetic stickers can be easily decorated at home without mounting. Paper-cut mounting can also be innovative. At present, the traditional ocean paper-cut is mainly mounted with photo frames and picture frames, which are exquisite, but there are also some heavy drawbacks. Paper-cut mounting can also use scrolls, fans, etc., to overcome the shortcomings of not easy to carry.

In addition, artists should learn from paper-cut works with different styles at home and abroad. From the North to the South, from the Central Plains to the border areas, from the Han nationality to the minority nationalities, from China to foreign countries, there are many different styles of paper-cut works and schools at home and abroad. Domestic paper-cut such as Shanxi paper-cut, Yangzhou paper-cut, Wenzhou Yueqing paper-cut, Wenling ocean paper-cut and so on, through understanding the domestic and foreign paper-cut artistic style and skills to make up for the shortcomings of Zhoushan ocean paper-cut, providing continuous power for the innovation and development of ocean paper-cut.

\section{CONCLuSion}

Zhoushan ocean folk paper-cut art is an important intangible cultural heritage of Zhoushan Islands with strong island regional characteristics. It has experienced twists and turns in the process of its inheritance and development. At present, it has also ushered in a new development situation. With the effective interaction of government, market and inheritors, Zhoushan folk ocean paper-cut will get out of the predicament and be promoted and developed, and will be better inherited with the development of the region. At the same time, Zhoushan folk ocean paper-cut art can also bring new development to the economy, culture, education and other aspects of the region.

\section{REFERENCE}

[1] Lv Shengzhong. Chinese Folk Paper-cut [M]. Changsha: Hunan Fine Arts Press, 1999

[2] Qiao Xiaoguang. Chinese Folk Art [M]. Hunan: Hunan Arts Press, 2011

[3] Bi Xuling, Tang Meng. Development of China's Marine Culture and Marine Culture Industry [M]. Shanghai: Oriental Press, 2016.7

[4] Wang Ying, Yang Guangxi. Cultural Memory of "Intangible Cultural Heritage" of Island: Biography of Inheritors of Zhoushan Intangible Cultural Heritage [M]. Hangzhou: Zhejiang University of Industry and Commerce Press, 2014

[5] Feng Xiao. Protection of Intangible Cultural Heritage And Industrial Research and Development [M]. Beijing: People's Daily Press, 2016

[6] Yu Wenshan,Cheng Xinghong,Luo Xiaoxue. Research on The Inheritance and Development of Folk Paper-Cut Art in Fuyang [J]. Journal of Bengbu University, 2017, 6 (05): 43-48.

[7] Liu Heyong. On the Marine Culture of Zhoushan Islands [M]. Beijing: Marine Press, 2006

[8] Wen Lei, Wu Qiong, Fu Rong, Sun Xiaodong. Research on Intellectual Property Protection in the Industrialization of Intangible Cultural Heritage: Based on the Analysis of Folk paper-cut Art [J]. Economic Issues, 2017 (09): 76-80. 
[9] $\mathrm{Hu}$ Ye, Hu Chunsheng. Wenzhou Paper-cut [M]. Hangzhou: Zhejiang Photo Press, 2010

\section{AUTHORS' BIOGRAPHY}

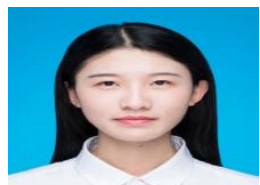

Yu Lihong, was born in Shaoxing, China in 1995. She is a currently a postgraduate at Zhejiang Ocean University, China, majoring in Rural Development. Her research interests include rural culture construction.

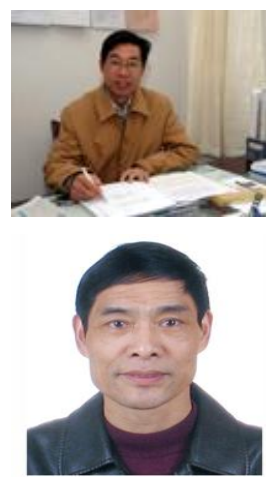

Song Qiuqian, was born in Zhoushan, China. He is a professor in the College of Teacher Education, College of Economics and Management, Zhejiang Ocean University, China.

Chen Shiding, was born in Zhoushan, China. He is a member of Chinese Folk Literature and Art Association and inheritor of ocean paper-cut intangible cultural heritage in Shengsi

Citation: Yu Lihong, Song Qiuqian, Chen Shiding. "A Study on the Current Situation and Protection of Zhoushan Folk Ocean Paper-Cut Art". International Journal of Humanities Social Sciences and Education (IJHSSE), vol. 6, no.6, 2019, pp. 24-29. doi: http://dx.doi.org/10.20431/2349-0381.0606004.

Copyright: () 2019 Authors. This is an open-access article distributed under the terms of the Creative Commons Attribution License, which permits unrestricted use, distribution, and reproduction in any medium, provided the original author and source are credited. 